\title{
Immune regulation mechanism of vitamin D level and IL-17/IL-17R pathway in Crohn's disease
}

\author{
YANLI XIA, HONGWEI CHEN, HONGLI XIAO, JING YANG, ZHIBIN LI, \\ YOUCHUN WANG, TIAN YANG and BAOYONG WANG \\ Department of Gastroenterology, Luoyang Central Hospital Affiliated to \\ Zhengzhou University, Luoyang, Henan 471000, P.R. China
}

Received September 21, 2018; Accepted February 8, 2019

DOI: $10.3892 /$ etm.2019.7389

\begin{abstract}
Immune regulation mechanism of vitamin D level and interleukin (IL)-17/IL-17 receptor (IL-17R) pathway in Crohn's disease was studied. Of 40 clean mature healthy rats, 10 rats were used as control group based on random number table, the remaining 30 rats to establish Crohn's disease rat models. After successful modeling, 30 rats were divided into model group, low-dose group and high-dose group with random number table. On the 1st day after modeling, rats in low-dose group were given a single dose of 1,750 IU of vitamin D, and rats in high-dose group a single dose of 7,500 IU of vitamin D. Changes in the condition of rats after modeling were observed and scored. Enzyme-linked immunosorbent assay was used for detecting IL-12, IL-17 and CXCL11 levels, western blotting for detecting IL-17R level, and flow cytometry for detecting Th1 cell and Th17 cell levels in the lamina propria of colon mucosa. Disease activity index scores were significantly lower in low-dose group and high-dose group of rats than those in model group $(\mathrm{P}<0.05)$. Those were significantly lower in high-dose group of rats than those in low-dose group $(\mathrm{P}<0.05)$. IL-17 and IL-17R levels were significantly lower in high-dose group of rats than those in low-dose group $(\mathrm{P}<0.05)$. Th1 cell level was significantly higher in high-dose group of rats than that in low-dose group $(\mathrm{P}<0.05)$, but Th17 cell level was lower than that in low-dose group $(\mathrm{P}<0.05)$. IL-12 levels were significantly higher in model group, low-dose group and highdose group of rats than those in control group $(\mathrm{P}<0.05)$. CXCL11 levels were significantly lower in model group, low-dose group and high-dose group of rats than those in control group $(\mathrm{P}<0.05)$. Vitamin D can effectively treat Crohn's disease, which may improve the chemotaxis and differentiation of Th1 cells by
\end{abstract}

Correspondence to: Dr Baoyong Wang, Department of Gastroenterology, Luoyang Central Hospital Affiliated to Zhengzhou University, 288 Zhongzhouzhong Road, Luoyang, Henan 471000, P.R. China

E-mail: wwy4rf@163.com

Key words: vitamin D level, IL-17, Crohn's disease, rat model inhibiting IL-17/IL-17R pathway, thereby improving immune function and reducing the severity of disease.

\section{Introduction}

Crohn's disease is a recurrent chronic inflammatory bowel disease based on genetic susceptibility, with environmental factors involved. It occurs mostly in young and middle-aged people with an incidence of approximately 1 in 200. Many patients with Crohn's disease experience feeble physical symptoms (such as emergency diarrhea, hemoproctia, vomiting, anorexia and lethargy) throughout their lives, seriously affecting their mental health and quality of life $(1,2)$. At present, the etiology and pathogenesis of Crohn's disease have not been fully studied, so there is a lack of specific treatment for it. In addition, it is often accompanied by recurrent complications such as intestinal obstruction and fistulization, so its medical expenses are also higher, which can be as high as 2 billion USD in the United States. Therefore, it is very important to find cost-effective drugs $(3,4)$.

Currently, many studies show that intestinal tissue damage is mainly caused by abnormalities in the immune response of intestinal mucosa with genetic susceptibility as a result of genetic and environmental factors $(5,6)$. In recent years, some studies have found that in addition to regulating calcium and phosphorus metabolism, vitamin D also regulates immune function. Studies report that vitamin D deficiency is prevalent in patients with Crohn's disease, correlated with the degree of disease activity $(7,8)$. Vitamin D has been currently reported to be able to alleviate the symptoms of Crohn's disease $(9,10)$, but its specific mechanism of action has not been fully studied. Secreted by Th17 cells, interleukin (IL)-17 exerts anti- and pro-inflammatory effects by binding to IL-17 receptor (IL-17R) (11). It has been reported that IL-17 can inhibit the differentiation of $\mathrm{CD}^{+} \mathrm{T}$ cells into $\mathrm{Th} 1$ cells and regulate immune function, and its expression level significantly increases in Crohn's disease (12).

It is hypothesized that the therapeutic effects of vitamin D may be related to IL-17/IL-17R pathway. Therefore, Crohn's disease rat models were established, to study the immune regulation mechanism of vitamin D level and IL-17/IL-17R pathway in Crohn's disease. 


\section{Materials and methods}

Study objects. A total of 40 clean mature healthy Sprague-Dawley (SD) rats were purchased from the Experimental Animal Center of Guangzhou University of Traditional Chinese Medicine (Guangdong, China; production license no. SCXK 2013-0034), with an average age of $43.6 \pm 1.7$ days and an average body weight of $235.3 \pm 7.6 \mathrm{~g}$. They were fed with ordinary nutrient feed (Jiangsu Synergy Pharmaceutical Bioengineering Co., Ltd., Jiangsu, China), with acidified drinking water that had a $\mathrm{pH}$ value between 2.5 and 3 after autoclaved sterilization, a feeding temperature of $17-21^{\circ} \mathrm{C}$ and a relative humidity of $45-65 \%$. They were fed separately in terrariums, with bedding changed regularly every morning and evening; ambient noise less than 85 decibels; ammonia concentration less than $20 \mathrm{PPm}$; 8-12 times of ventilation per hour; 1-2 times of nest changing, cleaning and disinfection every week; fluorescent lamp with a 12-h cycle of light. Ten rats were used as control group with random number table, the remaining 30 rats to establish Crohn's disease rat models. After successful modeling, 30 rats were divided into model group, low-dose group and high-dose group based on random number table.

This study was approved by the Ethics Committee of Luoyang Central Hospital Affiliated to Zhengzhou University (Luoyang, China).

Modeling methods. After fed for 7 days, all rats were fasted for $24 \mathrm{~h}$, routinely and disinfected in their abdomen. Then $10 \%$ chloral hydrate (Shanghai Shifeng Biotechnology Co., Ltd., Shanghai, China; $300 \mu \mathrm{g} / \mathrm{g}$ body weight) was intraperitoneally injected for anesthesia. A silicone catheter (Beijing Huakang Pumei Technology Co., Ltd., Beijing, China) was inserted into the colon, approximately $6-8 \mathrm{~cm}$ from the anus. The prepared trinitrobenzenesulfonic acid (TNBS; Shanghai Shifeng Biotechnology Co., Ltd.) solution (TNBS mixed with $40 \%$ ethanol solution at a ratio of 2:1) was perfused through a catheter at a dose of $150 \mathrm{mg} / \mathrm{kg}$, to establish Crohn's disease rat models. Rats in control group were perfused with an equal volume of saline. All rats were routinely fed after modeling operation. The day of the modeling operation was the 0 day.

Administration methods. On the 1st day after modeling, rats in control group and model group were routinely supplied with feed and drinking water, rats in low-dose group given a single dose of 1,750 IU of vitamin D (Shanghai Yihe Biotechnology Co., Ltd., Shanghai, China; item no. YH-011523S), and rats in high-dose group given a single dose of 7,500 IU of vitamin D. Rats in low-dose group and high-dose group were intragastrically fed and administered for consecutive 10 days. After 10 days of administration, relevant indicators were detected.

Observation indicators. Changes in the condition of rats after modeling were observed and scored based on the scoring standards of disease activity index. After 10 days of administration, the colon of rats was taken during operation. ELISA was used for detecting IL-12, IL-17 and CXCL11 levels in colon tissues, western blotting for detecting IL-17R level in colon tissues, flow cytometry for detecting Th1 cell and Th17 cell levels in the lamina propria of colon mucosa. Rats were sacrificed by acute blood loss. Specific operations referred to the instructions of reagents and instruments. Detection kits were purchased from Shanghai Jingkang Bioengineering Co., Ltd. (Shanghai, China).

Statistical analysis. SPSS version 19.0 (AsiaAnalytics, Formerly SPSS China, Shanghai, China) was used. Enumeration data were expressed as $[\mathrm{n}(\%)] \cdot \chi^{2}$ test was used for the comparison of ratio. Measurement data are expressed as mean \pm standard deviation. Student's t-test was used for comparison between the two groups, ANOVA for repeated measurement for comparison at different times in the group, and ANOVA for comparison among multiple groups with LSD test. $\mathrm{P}<0.05$ was considered to indicate a statistically significant difference.

\section{Results}

Disease activity index scores. There were significant differences in disease activity index scores among the four groups of rats $(\mathrm{P}<0.05)$. Disease activity index scores were significantly higher in model group, low-dose group and high-dose group of rats than those in control group $(\mathrm{P}<0.05)$, and were significantly lower in low-dose group and high-dose group of rats than those in model group $(\mathrm{P}<0.05)$, and were significantly lower in high-dose group of rats than those in low-dose group $(\mathrm{P}<0.05$; Fig. 1$)$.

Changes in body weight of rats. There were no statistical differences in body weight among the four groups of rats on the 0 and 1st day $(P>0.05)$, but there were statistical differences on the 3rd, 7th and 10th day $(\mathrm{P}<0.05)$. On the 3rd, 7th and 10th day, the body weight was lower in model group, low-dose group and high-dose group of rats than that in control group $(\mathrm{P}<0.05)$. The difference was higher in low-dose group and high-dose group of rats than that in model group $(\mathrm{P}<0.05)$, and was higher in high-dose group of rats than that in low-dose group $(\mathrm{P}<0.05)$. There were no statistical difference in the body weight in the group on the 0 and 1st day in the four groups of rats $(\mathrm{P}>0.05)$. From the 3 rd day, the body weight in control group, low-dose group and high-dose group of rats began to continuously increase $(\mathrm{P}<0.05)$. In model group of rats, the body weight was lower on the 3rd day than that on the 0 and 1 st day $(\mathrm{P}<0.05)$. There was no statistical difference on the 3rd, 7th and 10th day $(\mathrm{P}>0.05)$, which was lower than that on the 0 and 1st day ( $\mathrm{P}<0.05$; Table I and Fig. 2).

$I L-17$ and IL-17R levels. There were significant differences in IL-17 and IL-17R levels among the four groups of rats $(\mathrm{P}<0.05)$. IL-17 and IL-17R levels were significantly higher in model group, low-dose group and high-dose group of rats than those in control group $(\mathrm{P}<0.05)$, and were significantly lower in low-dose group and high-dose group of rats than those in model group $(\mathrm{P}<0.05)$, and were significantly lower in high-dose group of rats than those in low-dose group $(\mathrm{P}<0.05$; Fig. 3$)$.

Th1 and Th17 cell levels. There were significant differences in Th1 and Th17 cell levels among the four groups of rats $(\mathrm{P}<0.05)$. Th1 cell level was significantly lower in model group, low-dose group and high-dose group of rats than that 
Table I. Changes in body weight of rats.

\begin{tabular}{|c|c|c|c|c|c|c|}
\hline Day & Control group & Model group & Low-dose group & High-dose group & $\mathrm{t}$ & P-value \\
\hline 0 & $234.28 \pm 7.65$ & $228.47 \pm 7.58$ & $232.64 \pm 7.72$ & $231.15 \pm 7.42$ & 1.056 & 0.380 \\
\hline 1 & $236.36 \pm 7.46$ & $224.56 \pm 9.62$ & $233.75 \pm 14.43$ & $232.68 \pm 15.35$ & 1.752 & 0.174 \\
\hline 3 & $262.73 \pm 7.68$ & $213.69 \pm 10.43$ & $244.13 \pm 15.39$ & $254.47 \pm 15.41$ & 28.634 & $<0.001$ \\
\hline 7 & $320.12 \pm 8.33$ & $212.33 \pm 10.69$ & $276.42 \pm 15.58$ & $292.68 \pm 16.49$ & 117.462 & $<0.001$ \\
\hline 10 & $367.49 \pm 8.64$ & $201.48 \pm 10.72$ & $312.57 \pm 15.84$ & $334.37 \pm 16.56$ & 289.855 & $<0.001$ \\
\hline
\end{tabular}

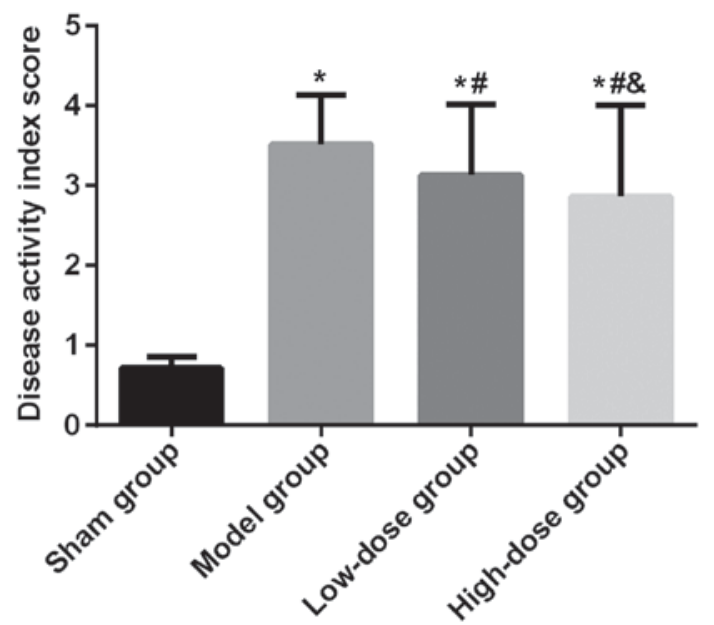

Figure 1. Disease activity index scores. Disease activity index scores were significantly higher in model group, low-dose group and high-dose group of rats than those in control group $(\mathrm{P}<0.05)$, and were significantly lower in low-dose group and high-dose group of rats than those in model group $(\mathrm{P}<0.05)$, and were significantly lower in high-dose group of rats than those in low-dose group $(\mathrm{P}<0.05)$. *compared to control group, $\mathrm{P}<0.05$; ${ }^{\#} \mathrm{com}-$ pared to model group, $\mathrm{P}<0.05 ;{ }^{\&}$ compared to low-dose group, $\mathrm{P}<0.05$.

in control group $(\mathrm{P}<0.05)$, but Th17 cell level was higher than that in control group $(\mathrm{P}<0.05)$. Th1 cell level was significantly higher in low-dose group and high-dose group of rats than that in model group $(\mathrm{P}<0.05)$, but Th17 cell level was lower than that in model group $(\mathrm{P}<0.05)$. Th1 cell level was significantly higher in high-dose group of rats than that in low-dose group $(\mathrm{P}<0.05)$, but Th17 cell level was lower than that in low-dose group (Fig. 4).

IL-12 and CXCL11 levels. There were significant differences in IL-12 and CXCL11 levels among the four groups of rats $(\mathrm{P}<0.05)$. IL-12 levels were significantly higher in model group, lowdose group and highdose group of rats than those in control group $(\mathrm{P}<0.05)$. CXCL11 levels were significantly lower in model group, low-dose group and high-dose group of rats than those in control group $(\mathrm{P}<0.05)$. IL-12 levels were significantly lower in low-dose group and high-dose group of rats than those in model group $(\mathrm{P}<0.05)$. CXCL11 levels were significantly higher in lowdose group and highdose group of rats than those in model group $(\mathrm{P}<0.05)$. IL-12 level was significantly lower in highdose group of rats than those in lowdose group $(\mathrm{P}<0.05)$. CXCL11 level was significantly higher in highdose group of rats than those in lowdose group $(\mathrm{P}<0.05$; Fig. 5).

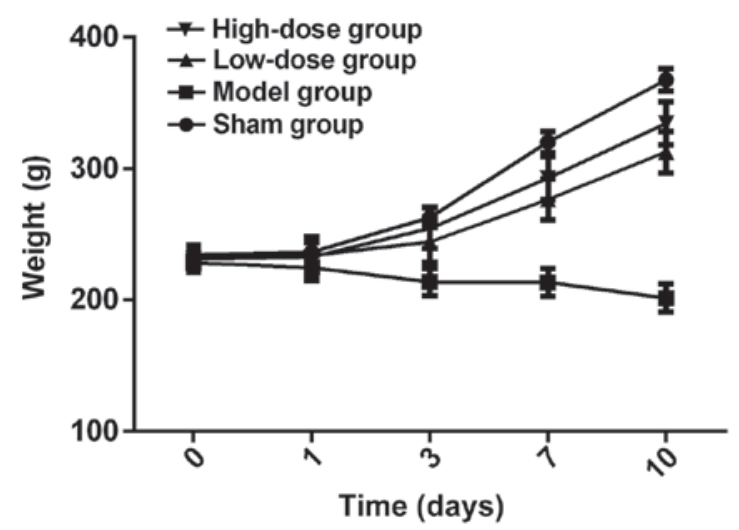

Figure 2. Changes in body weight of rats. There were no statistical differences in body weight among the four groups of rats on the 0 and 1st day $(\mathrm{P}>0.05)$. From the 3rd day, the body weight in control group, low-dose group and high-dose group of rats began to continuously to increase $(\mathrm{P}<0.05)$. In model group of rats, the body weight was lower on the 3 rd day than that on the 0 and 1 st day $(\mathrm{P}<0.05)$. There was no statistical difference on the 3rd, 7th and 10th day ( $P>0.05)$, which was lower than that on the 0 and 1 st day $(\mathrm{P}<0.05)$.

\section{Discussion}

Crohn's disease occurs mostly in developed countries, with lower incidence in developing countries, but its incidence is also rising with the development of economy (13). At present, Crohn's disease lacking effective treatment cannot be completely cured, thus increasing attention has been paid to it $(3,4)$. Vitamin D deficiency is common in patients with Crohn's disease, and vitamin D supplementation can effectively improve their quality of life and inflammatory response level $(9,10)$. Currently, the mechanism of action of vitamin D to alleviate the symptoms of Crohn's disease is not completely clear. Few reports exist on vitamin D and IL-17/IL-17R in Crohn's disease. Studies have reported (14) that vitamin D can reduce $\mathrm{CD}^{+}$cell level with positive IL-17 expression in psoriasis patients and improve their clinical symptoms. It has also (15) been reported that it can inhibit $\mathrm{T}$ cell proliferation, and reduce IL-17 level in inflammatory bowel disease and scleremus models. It is hypothesized that vitamin D may exert its therapeutic effects on Crohn's disease by acting on IL-17/IL-17R pathway.

In this study, the classical TNBS-induced Crohn's disease was used to replicate the pathological model of rats. After induction, rats in model group showed obvious symptoms of Crohn's disease, such as diarrhea, bloody stool, significant decrease in body weight, and increased disease activity index 
A

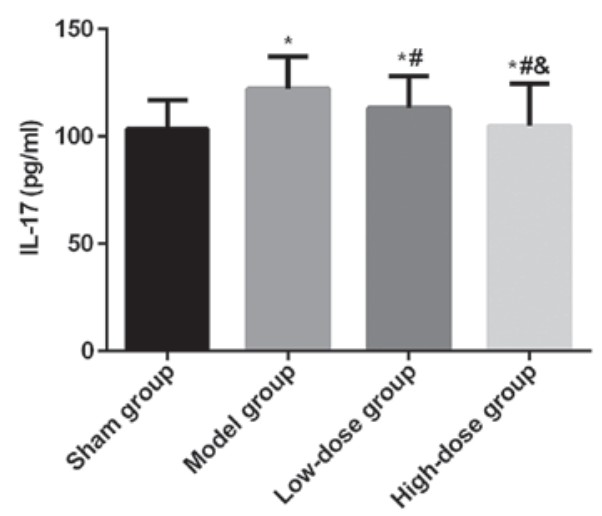

B

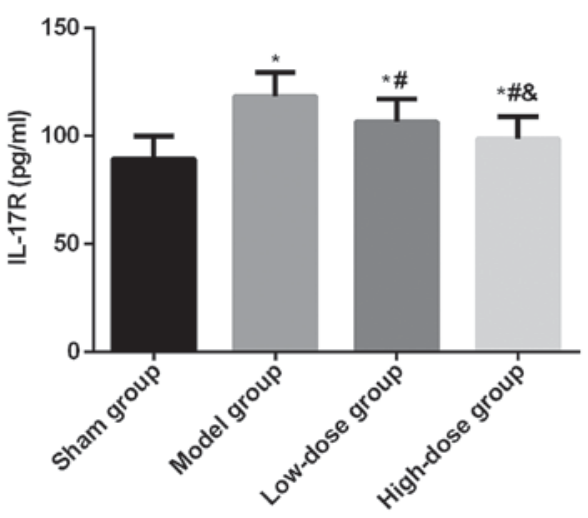

Figure 3. (A) Changes in IL-17 and (B) IL-17R levels. IL-17 and IL-17R levels were significantly higher in model group, low-dose group and high-dose group of rats than those in control group $(\mathrm{P}<0.05)$. They were significantly lower in low-dose group and high-dose group of rats than those in model group $(\mathrm{P}<0.05)$. They were significantly lower in high-dose group of rats than those in low-dose group $(\mathrm{P}<0.05)$. " compared to control group, $\mathrm{P}<0.05$; ${ }^{*}$ compared to model group, $\mathrm{P}<0.05 ;{ }^{*}$ compared to low-dose group, $\mathrm{P}<0.05$.

A

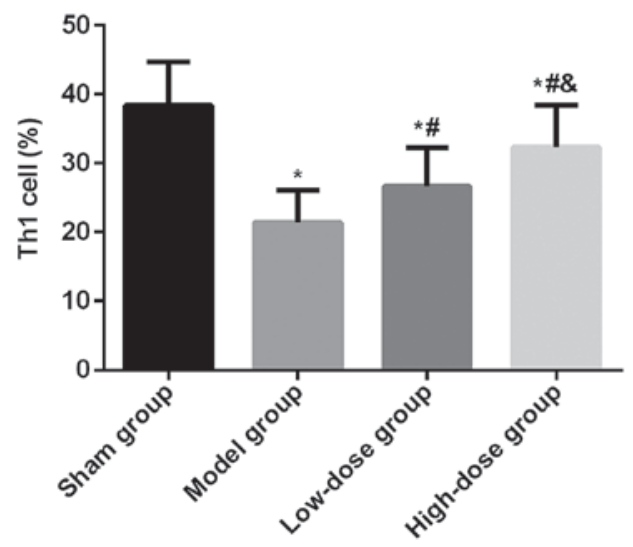

B

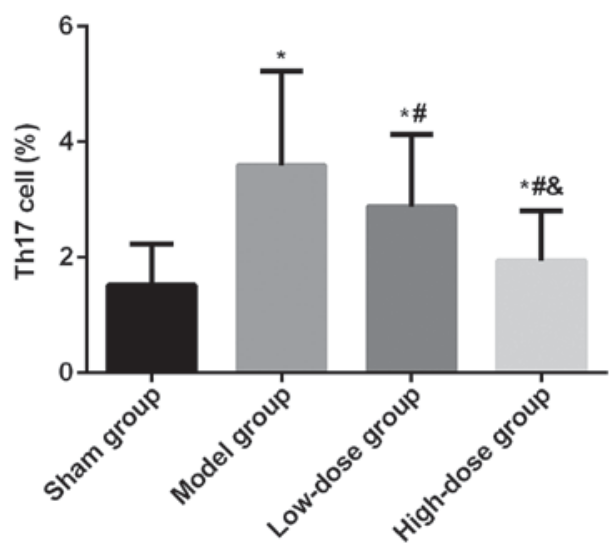

Figure 4. (A) Changes in Th1 cell and (B) Th17 cell levels. Th1 cell level was significantly lower in model group, low-dose group and high-dose group of rats than that in control group $(\mathrm{P}<0.05)$, but Th17 cell level was higher than that in control group $(\mathrm{P}<0.05)$. Th1 cell level was significantly higher in low-dose group and high-dose group of rats than that in model group $(\mathrm{P}<0.05)$, but Th17 cell level was lower than that in model group $(\mathrm{P}<0.05)$. Th1 cell level was significantly higher in high-dose group of rats than that in low-dose group $(\mathrm{P}<0.05)$, but Th17 cell level was lower than that in low-dose group $(\mathrm{P}<0.05)$. ${ }^{*}$ compared to control group, $\mathrm{P}<0.05 ;{ }^{*}$ compared to model group, $\mathrm{P}<0.05 ;{ }^{\&}$ compared to low-dose group, $\mathrm{P}<0.05$.

A

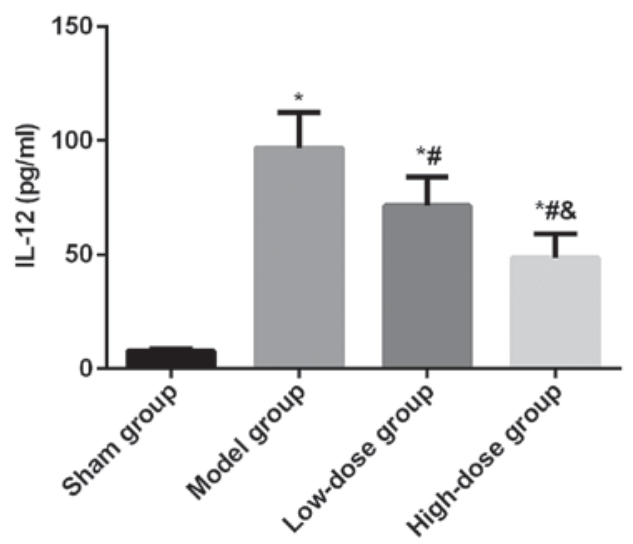

B

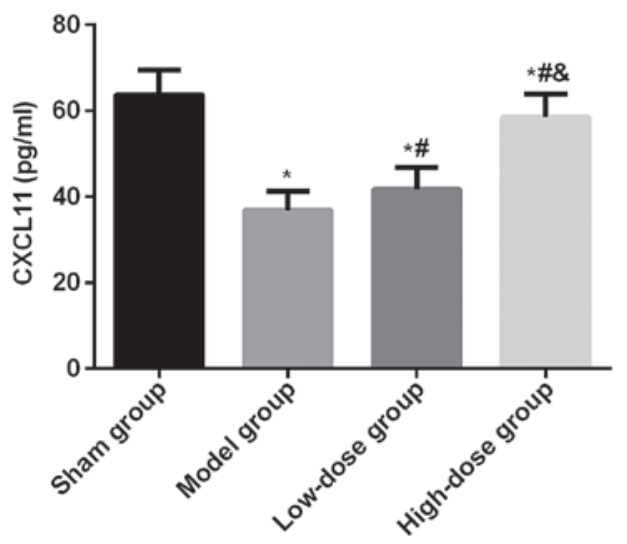

Figure 5. (A) Changes in IL-12 and (B) CXCL11 levels. IL-12 and CXCL11 levels were significantly higher in model group, low-dose group and high-dose group of rats than those in control group $(\mathrm{P}<0.05)$. CXCL11 levels were significantly lower in model group, low-dose group and high-dose group of rats than those in control group $(\mathrm{P}<0.05)$. IL-12 levels were significantly lower in low-dose group and high-dose group of rats than those in model group $(\mathrm{P}<0.05)$. CXCL11 levels were significantly higher in low-dose group and high-dose group of rats than those in model group ( $\mathrm{P}<0.05)$. IL-12 level was significantly lower in high-dose group of rats than those in low-dose group $(\mathrm{P}<0.05)$. CXCL11 level was significantly higher in high-dose group of rats than those in low-dose group $(\mathrm{P}<0.05)$. " compared to control group, $\mathrm{P}<0.05$; " compared to model group, $\mathrm{P}<0.05$; ${ }^{\star}$ compared to low-dose group, $\mathrm{P}<0.05$. 
with the increased number of stimulation, suggesting successful modeling in the experiment. Patients with Crohn's disease also have osteoporosis (16). For adult patients with osteoporosis, the recommended daily supplemental dose is 200 IU, and that of the elderly can be increased to 400-800 IU. However, the American Endocrine Association guidelines suggest that patients with Crohn's disease have a malabsorption of vitamin $\mathrm{D}$, so their daily supplemental dose should be more than 2-3 times patients of the same age, with a recommended daily dose of 1,500-2,000 IU. Therefore, combined with experimental conditions, the lowest dose of vitamin D was set to $1,750 \mathrm{IU}$, and the highest dose to 7,500 IU in this experiment (17). The results of this study showed that after the 1st day of treatment, low-dose group and high-dose group of rats showed significant decrease in the disease activity index, and the body weight began to increase when compared to model group. High-dose group of rats showed more significant decrease and increase when compared to low-dose group. It is indicated that the condition of rats in low-dose group and high-dose group was improved, while the body weight in model group of rats continued to decrease. In related research reports, vitamin D is effective in the treatment of Crohn's disease $(9,10)$, and the cyclic 25-hydroxy-vitamin D level is negatively correlated with the degree of Crohn's disease (18). Studies have reported that the oral high-dose of 10,000 IU of vitamin D3 per day can significantly increase the serum 25-hydroxy-vitamin D level, reducing clinical recurrence rate. Vitamin D3 is one of the manifestations of vitamin D in the human body (10). These studies also confirm our results, further affirming the therapeutic effects of vitamin D on Crohn's disease.

In order to verify our hypothesis, IL-17, IL-17R, IL-12 and CXCL11 levels in colon tissues, and Th1 cell and Th17 cell levels in the lamina propria of colon mucosa were analyzed. IL-17 is the most representative in the IL-17 family. By binding to IL-17R, it inhibits the increase of Th1 cell level, exerting anti-inflammatory effects, and promotes the expression of Th17 cells and neutrophil chemotactic factors, exerting pro-inflammatory effects. This is also one of the immune regulation mechanisms of IL-17 $(19,20)$. IL-12 is one of the most important regulators of Th0 cells differentiating into Th1 cells (21), and CXCL11 is a chemokine of Th1 cells (22). The results of this study showed that after 10 days of administration, model group of rats showed increased IL-17, IL-17R and Th17 levels in colon tissues, but showed significantly increased IL-12 and decreased CXCL11 levels when compared to control group. Low-dose group and high-dose group of rats showed significantly decreased IL-17, IL-17R and Th17 levels in colon tissues, but showed significantly decreased IL-12 and decreased CXCL11 levels when compared to model group. The increase or decrease in high-dose group of rats was more significant than that in low-dose group. The results validate the inhibitory effects of IL-17 on Th1, which also confirm to some extent our hypothesis. In Crohn's disease models, IL-17 can inhibit the chemotaxis and differentiation of Th1 cells, which may break Th1/Th2 cell balance, thereby leading to abnormal immune response and colonic tissue damage (23). Vitamin D can alleviate this breaking of balance, playing a therapeutic role in Crohn's disease.

There are still some shortcomings in this study. At present, there is no way to completely simulate the clinical features of Crohn's disease. TNBS-induced rat models mainly cause changes in Th1 cells, which may lead to some offset in the analysis of result. Therefore, the results of this study still require more animal models and large clinical data for further verification.

In summary, vitamin D can effectively treat Crohn's disease, which may improve the chemotaxis and differentiation of Th1 cells by inhibiting IL-17/IL-17R pathway, thereby improving immune function and reducing the severity of disease.

\section{Acknowledgements}

Not applicable.

\section{Funding}

No funding was received.

\section{Availability of data and materials}

The datasets used and/or analyzed during the current study are available from the corresponding author on reasonable request.

\section{Authors' contributions}

YX wrote the manuscript. YX, JY and ZL observed and scored the changes of rates after modeling. $\mathrm{HC}$ and $\mathrm{HX}$ detected indicators. YW, TY and BW were responsible for statistical analysis. All authors read and approved the final manuscript.

\section{Ethics approval and consent to participate}

This study was approved by the Ethics Committee of Luoyang Central Hospital Affiliated to Zhengzhou University (Luoyang, China).

\section{Patient consent for publication}

Not applicable.

\section{Competing interests}

The authors declare that they have no competing interests.

\section{References}

1. Chuang LS, Villaverde N, Hui KY, Mortha A, Rahman A, Levine AP, Haritunians T, Evelyn Ng SM, Zhang W, Hsu NY, et al: A frameshift in CSF2RB predominant among Ashkenazi jews increases risk for Crohn's disease and reduces monocyte signaling via GM-CSF. Gastroenterology 151: 710-723.e2, 2016.

2. Ananthakrishnan AN, Huang H, Nguyen DD, Sauk J, Yajnik V and Xavier RJ: Differential effect of genetic burden on disease phenotypes in Crohn's disease and ulcerative colitis: Analysis of a North American cohort. Am J Gastroenterol 109: 395-400, 2014.

3. Gevers D, Kugathasan S, Denson LA, Vázquez-Baeza Y, Van Treuren W, Ren B, Schwager E, Knights D, Song SJ, Yassour M, et al: The treatment-naive microbiome in new-onset Crohn's disease. Cell Host Microbe 15: 382-392, 2014.

4. Cleynen I, Boucher G, Jostins L, Schumm LP, Zeissig S, Ahmad T, Andersen V, Andrews JM, Annese V, Brand S, et al: International inflammatory bowel disease genetics consortium: Inherited determinants of Crohn's disease and ulcerative colitis phenotypes: A genetic association study. Lancet 387: 156-167, 2016. 
5. Feagan BG, Sandborn WJ, Gasink C, Jacobstein D, Lang Y, Friedman JR, Blank MA, Johanns J, Gao LL, Miao Y, et al: UNITI-IM-UNITI Study Group: Ustekinumab as induction and maintenance therapy for Crohn's disease. N Engl J Med 375: 1946-1960, 2016.

6. De Cruz P, Kamm MA, Hamilton AL, Ritchie KJ, Krejany EO, Gorelik A, Liew D, Prideaux L, Lawrance IC, Andrews JM, et al: Crohn's disease management after intestinal resection: A randomised trial. Lancet 385: 1406-1417, 2015.

7. Del Pinto R, Pietropaoli D, Chandar AK, Ferri C and Cominelli F: Association between inflammatory bowel disease and vitamin D deficiency: A systematic review and meta-analysis. Inflamm Bowel Dis 21: 2708-2717, 2015.

8. Suibhne TN, Cox G, Healy M, O'Morain C and O'Sullivan M: Vitamin D deficiency in Crohn's disease: Prevalence, risk factors and supplement use in an outpatient setting. J Crohn's Colitis 6: $182-188,2012$.

9. Raftery T, Martineau AR, Greiller CL, Ghosh S, McNamara D, Bennett K, Meddings J and O'Sullivan M: Effects of vitamin D supplementation on intestinal permeability, cathelicidin and disease markers in Crohn's disease: Results from a randomised double-blind placebo-controlled study. United European Gastroenterol J 3: 294-302, 2015.

10. Narula N, Cooray M, Anglin R, Muqtadir Z, Narula A and Marshall JK: Impact of high-dose vitamin D3 supplementation in patients with Crohn's disease in remission: A pilot randomized double-blind controlled study. Dig Dis Sci 62: 448-455, 2017.

11. Monin L, Gudjonsson JE, Childs EE, Amatya N, Xing X, Verma AH, Coleman BM, Garg AV, Killeen M, Mathers A, et al: MCPIP1/regnase-1 restricts IL-17A- and IL-17C-dependent skin inflammation. J Immunol 198: 767-775, 2017.

12. Chen K, Eddens T, Trevejo-Nunez G, Way EE, Elsegeiny W, Ricks DM, Garg AV, Erb CJ, Bo M, Wang T, et al: IL-17 receptor signaling in the lung epithelium is required for mucosal chemokine gradients and pulmonary host defense against K. pneumoniae. Cell Host Microbe 20: 596-605, 2016.

13. Monteleone G, Neurath MF, Ardizzone S, Di Sabatino A Fantini MC, Castiglione F, Scribano ML, Armuzzi A, Caprioli F, Sturniolo GC, et al: Mongersen, an oral SMAD7 antisense oligonucleotide, and Crohn's disease. N Engl J Med 372: 1104-1113, 2015.

14. Dyring-Andersen B, Bonefeld CM, Bzorek M, Løvendorf MB, Lauritsen JP, Skov L and Geisler C: The Vitamin D analogue calcipotriol reduces the frequency of $\mathrm{CD}^{+} \mathrm{IL}-17^{+} \mathrm{T}$ cells in psoriasis lesions. Scand J Immunol 82: 84-91, 2015.

15. Cantorna MT, Snyder L, Lin YD and Yang L: Vitamin D and 1,25(OH)2D regulation of T cells. Nutrients 7: 3011-3021, 2015.
16. Gomollón F, Dignass A, Annese V, Tilg H, Van Assche G, Lindsay JO, Peyrin-Biroulet L, Cullen GJ, Daperno M, Kucharzik T, et al: ECCO: 3rd European evidence-based consensus on the diagnosis and management of Crohn's disease 2016: Part 1: Diagnosis and medical management. J Crohn's Colitis 11: 3-25, 2017.

17. Gharib H, Papini E, Garber JR, Duick DS, Harrell RM, Hegedüs L, Paschke R, Valcavi R and Vitti P; AACE/ACE/AME Task Force on Thyroid Nodules: American Association of Clinical Endocrinologists, American College of Endocrinology, and Associazione Medici Endocrinologi Medical Guidelines for Clinical Practice for the Diagnosis and Management of Thyroid Nodules - 2016 Update. Endocr Pract 22: 622-639, 2016.

18. Sadeghian M, Saneei P, Siassi F and Esmaillzadeh A: Vitamin D status in relation to Crohn's disease: Meta-analysis of observational studies. Nutrition 32: 505-514, 2016.

19. Maxwell JR, Zhang Y, Brown WA, Smith CL, Byrne FR, Fiorino M, Stevens E, Bigler J, Davis JA, Rottman JB, et al: Differential roles for interleukin-23 and interleukin-17 in intestinal immunoregulation. Immunity 43: 739-750, 2015.

20. Kim J, Bissonnette R, Lee J, Correa da Rosa J, Suárez-Fariñas M, Lowes MA and Krueger JG: The spectrum of mild to severe psoriasis vulgaris is defined by a common activation of IL-17 pathway genes, but with key differences in immune regulatory genes. J Invest Dermatol 136: 2173-2182, 2016

21. Marchant A, Amedei A, Azzurri A, Vekemans J, Benagiano M, Tamburini C, Lienhardt C, Corrah T, McAdam KP, Romagnani S, et al: Polarization of PPD-specific T-cell response of patients with tuberculosis from Th0 to Th1 profile after successful antimycobacterial therapy or in vitro conditioning with interferon-alpha or interleukin-12. Am J Respir Cell Mol Biol 24: 187-194, 2001.

22. Liu Z, Wang M, Zhou S, Ma J, Shi Y, Peng J, Hou M and Guo C: Pulsed high-dose dexamethasone modulates Th1-/Th2-chemokine imbalance in immune thrombocytopenia. J Transl Med 14: 301, 2016.

23. Brand S: Crohn's disease: Th1, Th17 or both? The change of a paradigm: new immunological and genetic insights implicate Th17 cells in the pathogenesis of Crohn's disease. Gut 58: 1152-1167, 2009

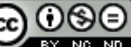

This work is licensed under a Creative Commons Attribution-NonCommercial-NoDerivatives 4.0 International (CC BY-NC-ND 4.0) License. 